\title{
Adjusting the cation and anion nature in ionic liquids used for the growth control of nanoparticles of organic conductors
}

Dominique de Caro $^{\mathrm{a}, \mathrm{b}^{*}}$, Christophe Faulmann ${ }^{\mathrm{a}, \mathrm{b}}$, Lydie Valade ${ }^{\mathrm{a}, \mathrm{b}^{*}}$, Kane Jacob ${ }^{\mathrm{a}, \mathrm{b}}$, Benoit Cormary ${ }^{\mathrm{a}, \mathrm{b}}$

${ }^{a}$ CNRS; LCC (Laboratoire de Chimie de Coordination); 205, route de Narbonne, F-31077

Toulouse, France

${ }^{\mathrm{b}}$ Université Fédérale de Toulouse; UPS, INPT; LCC; F-31062 Toulouse, France

dominique.decaro@lcc-toulouse.fr, lydie.valade@lcc-toulouse.fr

Keywords: organic conductor, superconductor, nanoparticle, electrocrystallization, ionic liquid. Ionic liquids are used for controlling the growth of organic conductors as nanoparticles. We review the conditions of preparation of nanoparticles of conductors derived from tetrathiafulvalene (TTF), tetramethyltetraselenafulvalene (TMTSF) and bis-ethylenedithiotetrathiafulvalene (BEDT-TTF). They are prepared by electrocrystallization using imidazolium or ammonium-based ionic liquids supporting electrolyte in which the cation plays the role of growth controller and the anion enters the composition of the expected organic conductor. Stable suspensions of nanoparticles are obtained in one case, a valuable characteristic for potential applications in electronic devices.

\section{Introduction}

Among the families of organic conductors and superconductors is the fractional oxidation state compounds of general formula $\mathrm{D}^{\mathrm{n}+} \mathrm{X}^{-} \mathrm{n}$, where $\mathrm{D}$ is an organic molecule such as tetrathiafulvalene (TTF), tetramethyltetraselenafulvalene (TMTSF) or bis-ethylenedithiotetrathiafulvalene (BEDT-TTF) (Figure 1), associated with a $\mathrm{X}^{-}$anion as $\mathrm{Cl}^{-}, \mathrm{Br}^{-}, \mathrm{PF}_{6}^{-}$or $\left[\mathrm{Cu}(\mathrm{SCN})_{2}\right]^{-}[1]$. This article will analyze the conditions of preparation of nanoparticles of the four conductors $\mathrm{TTFCl}_{0.77}, \mathrm{TTFBr}_{0.59}, \mathrm{BEDT}$ $\mathrm{TTFCl}_{0.66}$ and (BEDT-TTF) ${ }_{2} \mathrm{Br}$, and of the superconductor (TMTSF) ${ }_{2} \mathrm{PF}_{6}$, and apply them to the preparation of the first nanoparticles of the superconductor $(\mathrm{BEDT}-\mathrm{TTF})_{2}\left[\mathrm{Cu}(\mathrm{SCN})_{2}\right]$.

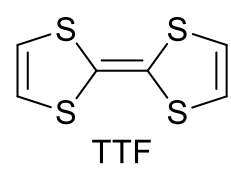<smiles>CC1=C(C)[Se]C(=C2[Se]C(C)=C(C)[Se]2)[Se]1</smiles><smiles>C1CSC2=C(S1)SC(=C1SC3=C(SCCS3)S1)S2</smiles>

BEDT-TTF

Figure 1. Molecular structures of tetrathiafulvalene (TTF), tetramethyltetraselenafulvalene (TMTSF) and bisethylenedithiotetrathiafulvalene (BEDT-TTF).

These salts are obtained by oxidation of the organic molecule $\mathrm{D}$, in the presence of a second reactive species affording the $\mathrm{X}^{-}$anion. The widest applied technique to grow single 
crystals of fractional oxidation state compounds is the electrocrystallization technique $[2,3]$. Galvanostatic conditions and low current densities are selected to grow large single crystals suitable for physical studies. As the room-temperature properties of organic conductors make them of interest in the field of organic electronics, these materials should be available in large quantities, reproducible morphologies and easy to process. Galvanostatic conditions of electrolysis afford low quantities of single crystals. Potentiostatic conditions could be used, but will not control the morphology of the materials as the growth rate is not constant. Poor solubility is an additional problem to process these materials by solution-based techniques such as inkjet or spray coating. This limitation can be overcome when the material is prepared as nanoparticles: for instance, colloidal solution of metals have been obtained by adding growth controllers to the reaction medium [4]. Therefore, we have chosen to investigate the preparation of organic conductors as nanoparticles by electrolysis, (i) in galvanostatic conditions to control the growth rate and (ii) in the presence of a imidazolium or ammonium-based ionic liquid (IL), a medium well-known to control the growth of materials as nanoparticles [5]. This is performed by replacing the cation of the supporting electrolyte, either by an ammonium salt containing long alkyl chains, or by an imidazolium cation (Figure 2). The originality of the procedure is that the electrolytic solution only contains the chemical species necessary to afford the expected phase. The IL plays a triple role: supporting electrolyte, source of anion of the expected phase and growth control of the morphology via the cation nature.

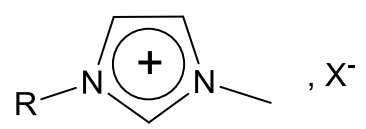

1-alkyl-3-methylimidazolium salt

$\mathrm{R}=$ butyl: $\mathrm{BMIM}^{+}, \mathrm{R}=$ ethyl: $\mathrm{EMIM}^{+}$ $\mathrm{X}^{-}=\mathrm{PF}_{6}^{-}, \mathrm{SCN}^{-}$

Figure 2. Imidazolium salts used for growth control of nanoparticles.

The application of this procedure was first successful to prepare nanoparticles of the $\mathrm{TTFCl}_{0.77}$ conductor [6]. We further confirmed its suitability for isolating nanoparticles of other molecular conductors as $\mathrm{TTFBr}_{0.59}$, (BEDTTTF $)_{2} \mathrm{Br}, \quad$ BEDT-TTFCl $_{0.66} \quad$ [7] and superconductors as (TMTSF) $)_{2} \mathrm{PF}_{6}$ [8]. The objective of this article is to analyze and review the conditions that favor the growth of the above molecular conductors as nanoparticles during their electrocrystallization. According to this analysis, the procedure is adapted to grow the first nanoparticles of the superconductor $(\mathrm{BEDT}-\mathrm{TTF})_{2}\left[\mathrm{Cu}(\mathrm{SCN})_{2}\right]$. The chemical and physical properties of the nanoparticles of this phase are compared to single crystals properties.

\section{Experimental part}

Materials

Organic molecules: TTF, TMTSF and BEDT-TTF are commercially available and used as received. 
Solvents: tetrahydrofuran, acetonitrile and methylene chloride are distilled and stored under argon before use.

Ionic liquids: $\mathrm{Me}(\mathrm{n}-\mathrm{Oct})_{3} \mathrm{NCl},(\mathrm{n}-\mathrm{Oct})_{4} \mathrm{~N}$ $\mathrm{Br}, \quad\left(\mathrm{BMIM}^{\mathrm{P} \mathrm{PF}_{6}}\right.$ and $(\mathrm{EMIM}) \mathrm{SCN}$ are commercially available. The $\left[\mathrm{Cu}(\mathrm{SCN})_{2}\right]^{-}$anion in $(\mathrm{BEDT}-\mathrm{TTF})_{2}\left[\mathrm{Cu}(\mathrm{SCN})_{2}\right]$ results from an in situ reaction between $\mathrm{CuSCN}$ and the $\mathrm{SCN}^{-}$ anion of the IL, (EMIM)SCN.

\section{Synthesis}

The electrolysis is conducted in a $\mathrm{H}$ shaped cell equipped with platinum wire electrodes, at room-temperature and under argon atmosphere. Both compartments contain $10 \mathrm{~mL}$ of a solution of the ionic liquid salt. The organic molecule is added to the anodic compartment. The molar ratio organic molecule/IL is $1 / 10$. In the case of $\mathrm{EMIM}\left[\mathrm{Cu}(\mathrm{SCN})_{2}\right],(\mathrm{EMIM}) \mathrm{SCN}$ is placed in both compartments and $\mathrm{CuSCN}$ is added to the anodic compartment. The anodic solution is stirred and a current density of $30 \mu \mathrm{A} / \mathrm{cm}^{2}$ is applied for about 3 days. The electrolysis duration can be reduced to an hour by applying higher current densities. Table 1 lists the combinations of organic molecules and IL salts investigated to prepare nanoparticles of the molecular conductors described in this article. The conducting phase grows on the anode as a black powder. The powder is not air sensitive and is collected from the anodic compartment by filtration and washed with the solvent used for the electrocrystallization (Table 1). The powder is finally dried under vacuum for typically $24 \mathrm{~h}$ and can be stored in air.

Table 1. Composition of the electrolytic solutions used for growing nanoparticles of organic conductors. ${ }^{*} \mathrm{Cu}(\mathrm{SCN})$ is added to the anodic solution to afford the $\left[\mathrm{Cu}(\mathrm{SCN})_{2}\right]^{-}$anion present in the expected organic conductor.

\begin{tabular}{|l|l|l|}
\hline $\begin{array}{l}\text { Organic } \\
\text { molecule }\end{array}$ & Ionic liquid salt & Solvent \\
\hline TTF & $\mathrm{Me}(n \text {-Oct })_{3} \mathrm{~N}^{+} \mathrm{Cl}^{-}$ & $\mathrm{CH}_{3} \mathrm{CN}$ \\
\hline TTF & $(n-\mathrm{Oct})_{4} \mathrm{~N}^{+} \mathrm{Br}^{-}$ & $\mathrm{CH}_{3} \mathrm{CN}$ \\
\hline TMTSF & $\mathrm{BMIM}^{+} \mathrm{PF}_{6}^{-}$ & $\mathrm{THF}$ \\
\hline BEDT-TTF & $\mathrm{Me}\left(n-\mathrm{Oct}_{3} \mathrm{~N}^{+} \mathrm{Cl}^{-}\right.$ & $\mathrm{CH}_{2} \mathrm{Cl}_{2}$ \\
\hline BEDT-TTF & $(n-\mathrm{Oct})_{4} \mathrm{~N}^{+} \mathrm{Br}^{-}$ & $\mathrm{CH}_{2} \mathrm{Cl}_{2}$ \\
\hline BEDT-TTF & $\mathrm{EMIM}^{+}(\mathrm{SCN})^{-*}$ & $\mathrm{CH}_{2} \mathrm{Cl}_{2}$ \\
\hline
\end{tabular}

\section{Characterization techniques}

X-ray diffraction data were collected with a Bruker D8 Advance diffractometer working in the Bragg-Brentano configuration $(\theta-\theta)$ using Ni filtered $\mathrm{CuK} \alpha$ radiation $(0.15418$ nm) and fitted with a SuperSpeed Vantec Detector. The powder pattern is compared to the pattern calculated from the single crystal structure using Mercury3.6 program.

The nanoparticle powder is dispersed in diethylether for TEM (transmission electronic microscopy) observation. The TEM samples are then prepared by evaporation of droplets of the suspension deposited on carbon-supported copper grids. The images were obtained from a JEOL 
Model JEM 1011 equipment operating at $100 \mathrm{kV}$.

AFM images were obtained from a AFM Smarts SPM 1000 (AIST-NT) equipment in the tapping mode (resonance frequency: 325 $\mathrm{kHz}$, force constant: $40 \mathrm{~N} / \mathrm{m}$ ) using back side Al-coated silicon cantilever tips (HQ:NSC15/ALB5 from MicroMash).

Raman spectroscopy is used to determine the charge of the organic molecule constitutive of the fractional oxidation state conductive materials. Bozio et al. have shown that the Raman shift of the $v_{C=C}\left(a_{g} v_{3}\right.$ mode) of the central double bond of the TTF molecule varies linearly with its charge [9]. This method also applies to the determination of the charge of TMTSF [10, 11] or BEDT-TTF [12]. Raman spectra of the isolated nanoparticle powders were recorded at $80 \mathrm{~K}$ using a DILOR $\mathrm{XY}$ micro-Raman (785 nm laser line). Results are reported in Table 2.

Table 2. Degree of charge ( $\rho)$ obtained from the Raman shift $\left(v_{\mathrm{C}=\mathrm{C}}\right)$ of the $\mathrm{C}=\mathrm{C}$ central band of the organic donor molecule and chemical composition of the corresponding electrochemically-grown nanoparticles.

\begin{tabular}{|l|l|l|l|}
\hline $\begin{array}{l}\text { Organic } \\
\text { molecule }\end{array}$ & $\begin{array}{l}\boldsymbol{v}_{\mathrm{C}=\mathrm{C}} \\
\mathbf{c m}^{-\mathbf{1}}\end{array}$ & $\boldsymbol{\rho}$ & Composition \\
\hline TTF & 1447 & 0.77 & $\mathrm{TTFCl}_{0.77}$ \\
\hline TTF & 1457 & 0.59 & $\mathrm{TTFBr}_{0.59}$ \\
\hline BEDT-TTF & 1464 & 0.66 & $($ BEDT-TTF)Cl \\
0.66 \\
\hline BEDT-TTF & 1471 & 0.50 & $(\text { BEDT-TTF })_{2} \mathrm{Br}$ \\
\hline TMTSF & 1460 & 0.50 & $(\text { TMTSF })_{2} \mathrm{PF}_{6}$ \\
\hline
\end{tabular}

\section{Results and discussion}

We have used imidazolium or ammonium-based ionic liquids as supporting electrolytes in the electrocrystallization of organic conductors as nanoparticles. IL are known to organize in arrays containing nanocavities where reactions can take place. This property has been successfully used to control the growth of metal or metal oxides [5], and magnetic molecular materials such as Prussian blue [13]. The stabilization of nanoparticles of these systems is explained by chemical interactions between the material and the IL, present as a shell around the particles. Prussian blue, metals, and metal oxides exhibit three-dimensional structures while organic conductors are more likely one-dimensional. Indeed, their crystal structures are made of stacks of the planar organic molecules interacting through $\pi$ and/or chalcogene interactions [1]. Consequently, most of organic conductors grow as needles or elongated platelets. For growing them as nanoparticles, the organization of IL is of interest to limit the particle growth. We have selected two families of IL able to create two types of interactions with the organic molecules of the material. Both families undergo ionic interactions with the organic molecules which are in a charged state within the final material. The ammonium salts containing long alkyl chains are also expected to act as shell layers avoiding aggregation of 
particles. Imidazolium salts offer possibilities of $\pi$-type interactions with the planar molecules. The anion associated to ammonium or imidazolium cations is chosen to afford the expected organic conductor.

In addition to these chemical reasons, the ILs that we have selected (Table 1) are all commercially available, a criterion that should be addressed for further large scale production of nanoparticles. Moreover, imidazolium ILs are among the more widely used for structuration purposes. However, in order to perform electrocrystallization of organic conductors in a IL medium, a number of conditions have to be adapted. We will explain these adaptations through the description of the nanoparticle growth of $\mathrm{TTFCl}_{0.77}, \mathrm{TTFBr}_{0.59}$, (TMTSF) ${ }_{2} \mathrm{PF}_{6}$, BEDT-TTFCl $_{0.66}$, (BEDT-TTF $)_{2} \mathrm{Br}$, and apply them to the synthesis of the first isolated nanoparticles of (BEDT-TTF $)_{2}\left[\mathrm{Cu}(\mathrm{SCN})_{2}\right]$.

Solvent: ILs can be used as the solvent medium in chemical or electrochemical reactions. For example, cyano-bridged magnetic nanoparticles were prepared using directly $(\mathrm{BMIM}) \mathrm{BF}_{4}$ as the solvent [13]. We could expect that, in addition to the role of supporting electrolyte, the selected IL could also be used as the solvent in the electrocrystallization of organic conductors. This was not possible because the solubility of neutral TTF, TMTSF and BEDT-TTF molecules in ILs is too low. Therefore, the same solvent as used for growing single crystal of the expected phase was chosen to dissolve both the organic molecule and the IL and obtain a homogeneous electrolytic solution.

Amount of ionic liquid: in the electrocrystallization of organic conductors as nanoparticles, the IL plays the role of the supporting electrolyte. The typical concentration ratio between the electroactive species and the supporting salt is generally set to $1 / 100$ when the electrolysis is conducted in potentiostatic conditions. This concentration ratio is often reduced to $1 / 10$ when galvanostatic conditions are applied, mainly to increase the concentration of the electroactive species [2].

Several organic molecule/IL concentration ratios were investigated. For example, the electrosynthesis of $\mathrm{TTFCl}_{0.77}$ was conducted using TTF/Me $(n-\mathrm{Oct})_{3} \mathrm{NCl}$ concentration ratios of $1 / 3$ and $1 / 10$. As evidenced by the TEM images of Figure 3, when the amount of IL is 3 times that of TTF, the isolated material contains only needles. The efficiency of the IL as growth controller becomes significant when its amount is at least 10 times that of TTF. In these conditions, TTFCl $_{0.77}$ grows as spherical nanoparticles exhibiting a mean diameter of $20 \mathrm{~nm}$. Therefore, reducing the IL concentration does not avoid the electrolysis to occur, but does not avoid either the material to grow as needles. On the other hand, when a sufficient amount of $\operatorname{Me}(n-$ $\mathrm{Oct})_{3} \mathrm{~N}^{+}$cations are present at the vicinity of the electrode, they control the crystallites growth. It appears that the $1 / 10$ ratio between the 
electroactive species and the electrolyte is convenient also when the electrolyte is of IL type. This ratio was applied in all further electrocrystallization.

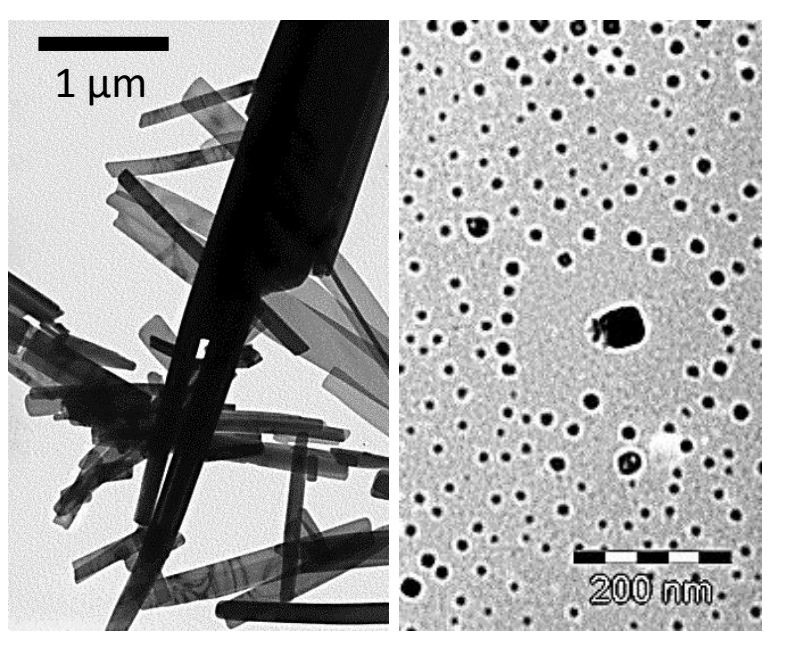

Figure 3. TEM images of $\mathrm{TTFCl}_{0.77}$ prepared by galvanostatic electrolysis of an acetonitrile solution of TTF and $\mathrm{Me}(n-\mathrm{Oct})_{3} \mathrm{NCl}$ in concentration ratio: (left) $1 / 3$, (right) $1 / 10$.

Stirring: we have shown that, in the electrocrystallization of NPs of organic conductors, IL salts play a similar role as a standard supporting electrolyte in term of support of the migration current. The IL concentration has to be at least 10 times that of the electroactive species for playing a structuring role during the growth process. ILs contain bulky cations compared to conventional supporting electrolytes. It was reported that during the electrochemical growth of cobalt NPs, the electrode was rapidly passivated by the bulky long chains of $(n-\mathrm{Oct})_{4} \mathrm{NBr}$ used as supporting electrolyte [14]. Vigorous stirring is necessary to favor the access of the electroactive species to the electrode.
Figure 4 shows the nanoparticles isolated in the case of $\mathrm{TTFBr}_{0.59}$, BEDTTTFCl $_{0.66}$ and (BEDT-TTF) $)_{2} \mathrm{Br}$ by combining the conditions described above: galvanostatic conditions to have a constant growth rate, amount of IL 10 times that of the electroactive species to have both support of the migration current and to limit the growth, and stirring to increase the access of the electroactive species to the electrode.
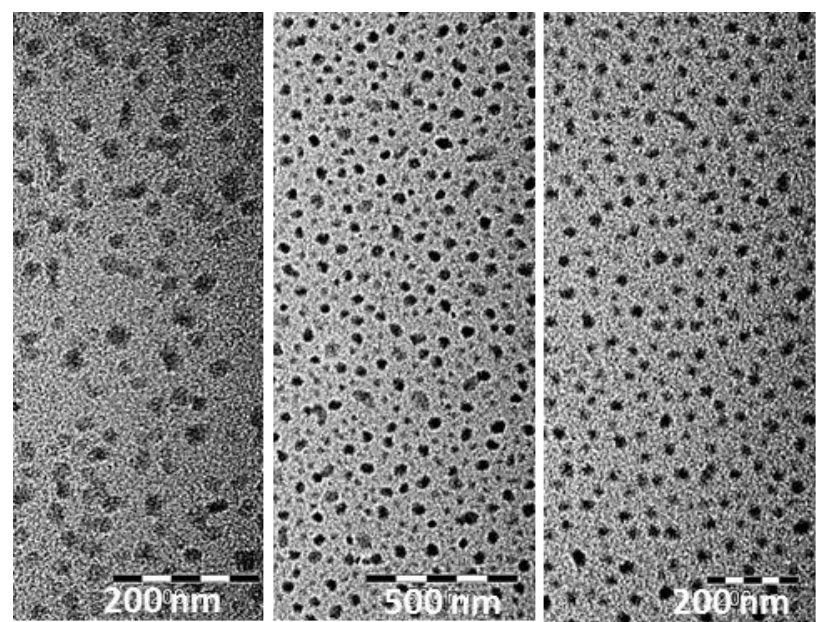

Figure 4. TEM images of nanoparticles of (from left to right) $\mathrm{TTFBr}_{0.59}, \mathrm{BEDT}-\mathrm{TTFCl}_{0.66}$ and (BEDT-TTF) ${ }_{2} \mathrm{Br}$, prepared by galvanostatic electrolysis of a solution of TTF or BEDT-TTF and the appropriate IL electrolyte and solvent (see Table 1) in a concentration ratio of $1 / 10$.

The mean size of the NPs is in the same range: $30 \mathrm{~nm}$ for $\mathrm{TTFBr}_{0.59}, 50 \mathrm{~nm}$ for BEDT$\mathrm{TTFCl}_{0.66}$ and $40 \mathrm{~nm}$ (BEDT-TTF) ${ }_{2} \mathrm{Br}$ [7]. Raman studies of the nanoparticle powders show that the use of ionic liquids as supporting electrolytes has no consequence on the chemical composition of the isolated materials (Table 2), which is that of single crystals. Although these analyses do not evidence the presence of IL on 
the surface of the NPs, it is clear that its presence in the electrochemical solution is necessary to control the growth of the organic conductors as nanoparticles.

High current densities: the application of galvanostatic conditions to the growth of organic conductors is preferred to potentiostatic conditions because it allows a constant growth rate. On the other hand, low current densities are accompanied with the production of low amounts of material. We have investigated the influence of the increase of current densities on the morphology of nanoparticles of (TMTSF) ${ }_{2} \mathrm{PF}_{6}$ with the aim to obtain a larger amount of material in a minimum of time.

Figure 5 shows that well-dispersed NPs are obtained in all conditions. The average size of NPs is not drastically modified. However, the dispersion in size is lower at the two highest current densities than at the lowest one. The NPs grown at $30 \mu \mathrm{A} / \mathrm{cm}^{2}$ have a size ranging from $30 \mathrm{~nm}$ to $50 \mathrm{~nm}$, while all particles are about $50 \mathrm{~nm}$ and $55 \mathrm{~nm}$ when grown at 600 and $1500 \mu \mathrm{A} / \mathrm{cm}^{2}$, respectively. The chemical composition of the NPs is not modified by high current density conditions (Table 2) [15]. This result demonstrates that homogeneous NPs can be produced in $1 \mathrm{~h} v s .3$ days, at least in small scale electrochemical cells. Larger scale experiments can now be envisioned as NPs have to be produced at gram scale for applications.
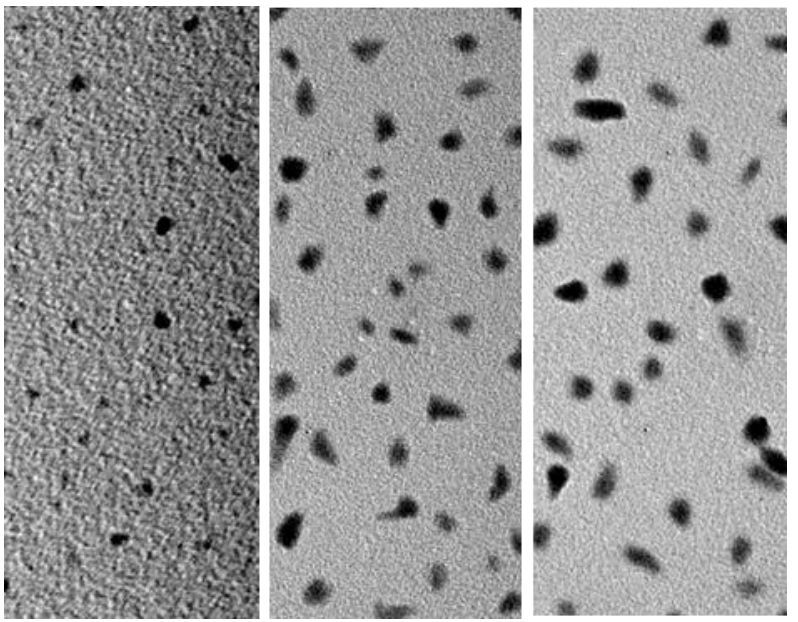

Figure 5. TEM images at the same magnification of nanoparticles of (TMTSF) $)_{2} \mathrm{PF}_{6}$, prepared by galvanostatic electrolysis of a $1 / 10$ solution of TMTSF and (BMIM)PF in THF at three current densities: 30, 600 and 1500 $\mu \mathrm{A} / \mathrm{cm}^{2}$ (from left to right). The corresponding electrolysis duration is 3 days, $3 \mathrm{~h} 50$ and $1 \mathrm{~h} 15$, respectively.

Synthesis of NPs of the (BEDT$\underline{\mathrm{TTF}})_{2}\left[\mathrm{Cu}(\mathrm{SCN})_{2}\right]$ phase: BEDT-TTF is known to form two phases of same stoichiometry but different structure with the $\left[\mathrm{Cu}(\mathrm{SCN})_{2}\right]^{-}$anion: the first one, $\kappa-(\mathrm{BEDT}-\mathrm{TTF})_{2}\left[\mathrm{Cu}(\mathrm{SCN})_{2}\right]$, is an ambient pressure superconductor $[16,17]$ with a superconducting transition temperature of $\mathrm{Tc}=$ $10.4 \mathrm{~K}$, while the second one, $\alpha$-(BEDT$\mathrm{TTF})_{2}\left[\mathrm{Cu}(\mathrm{SCN})_{2}\right]$ is metallic around room temperature and becomes semiconductive below about $200 \mathrm{~K}$ [18].

Taking into account the conditions of preparation of NPs described above, the electrosynthesis of NPs of (BEDT-TTF $)_{2}$ $\left[\mathrm{Cu}(\mathrm{SCN})_{2}\right]$ was conducted at $300 \mu \mathrm{A} / \mathrm{cm}^{2}$ for $14 \mathrm{~h}$ in dichloromethane and using (EMIM)SCN as the supporting electrolyte. The electrolytic solution is prepared at $70{ }^{\circ} \mathrm{C}$ to increase the 
solubility of BEDT-TTF. The electrolysis is conducted at room temperature. As for the electrosynthesis of single crystals, $\mathrm{Cu}(\mathrm{SCN})$ is added to the anodic solution to form the $\left[\mathrm{Cu}(\mathrm{SCN})_{2}\right]^{-}$anion. Figure $\mathbf{6}$ shows that roughly spherical NPs are obtained with a mean size of $10 \mathrm{~nm}$, smaller than the other studied phases.
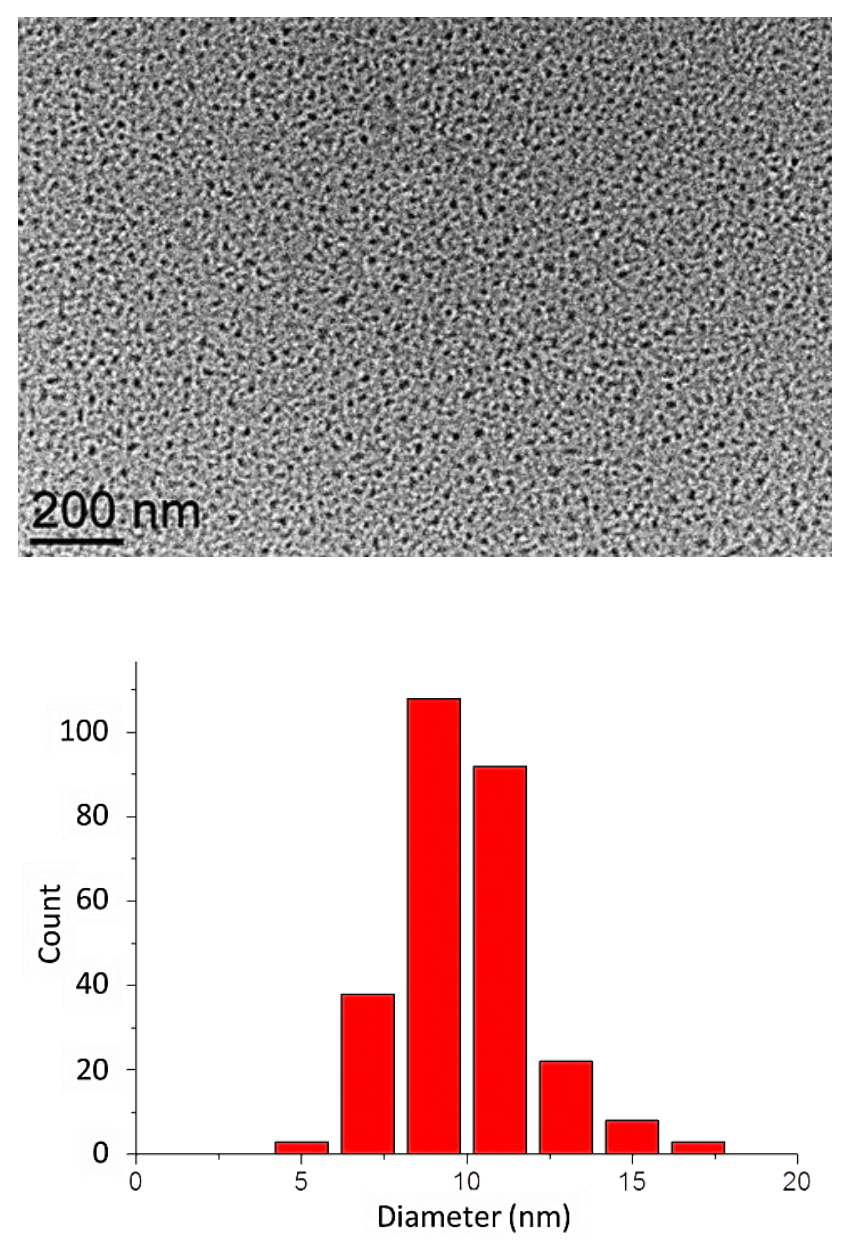

Figure 6. TEM image at $100 \mathrm{kV}$ and histogram of size of nanoparticles of (BEDT-TTF $)_{2}\left[\mathrm{Cu}(\mathrm{SCN})_{2}\right]$ deposited on a carbon-supported copper grid. The NPs are prepared by galvanostatic electrolysis of a 1/0.5/10 solution of BEDT$\mathrm{TTF} / \mathrm{CuSCN} /(\mathrm{EMIM}) \mathrm{SCN}$ in $\mathrm{CH}_{2} \mathrm{Cl}_{2}$ at $300 \mu \mathrm{A} / \mathrm{cm}^{2}$.

The size of the nanoparticles is confirmed by AFM observation (Figure 7). The $\mathrm{X}$-Ray diffraction pattern of the nanoparticles powder was compared to the pattern simulated using the structure of single crystals of the $\kappa$ $(\mathrm{BEDT}-\mathrm{TTF})_{2}\left[\mathrm{Cu}(\mathrm{SCN})_{2}\right]$ phase (Figure 8). The NPs pattern exhibits the more intense lines of the pattern of the $\kappa$-phase, although additional lines are observed and may indicate the simultaneous presence of the $\alpha$-(BEDT$\mathrm{TTF})_{2}\left[\mathrm{Cu}(\mathrm{SCN})_{2}\right]$ phase.
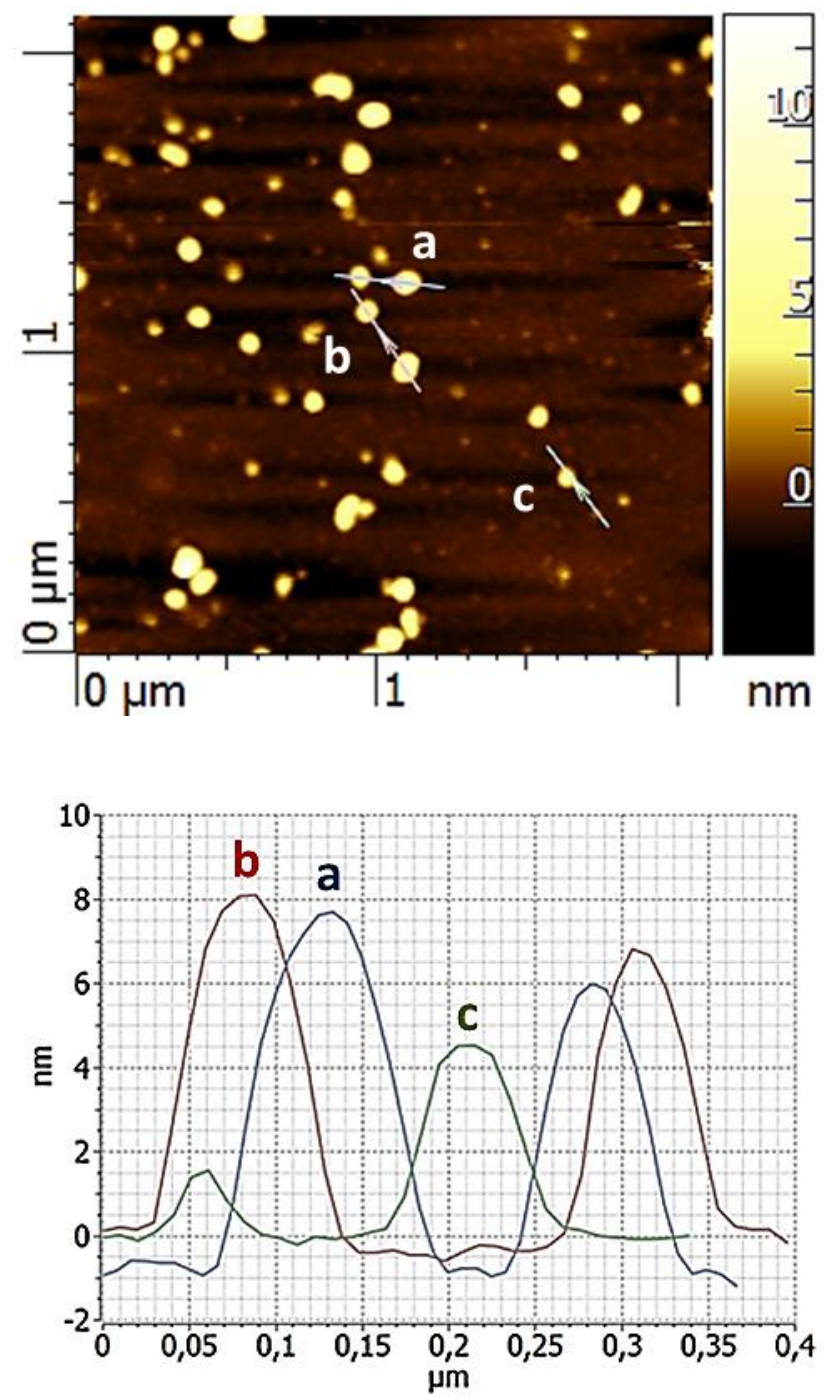

Figure 7. AFM image (tapping mode) of nanoparticles of $(\mathrm{BEDT}-\mathrm{TTF})_{2}\left[\mathrm{Cu}(\mathrm{SCN})_{2}\right]$. The graph shows the line profile analysis of the image where the $\mathrm{Y}$ axis is the height (diameter) of the nanoparticles and the $\mathrm{X}$ axis is the length of the line scanned by the tip. 


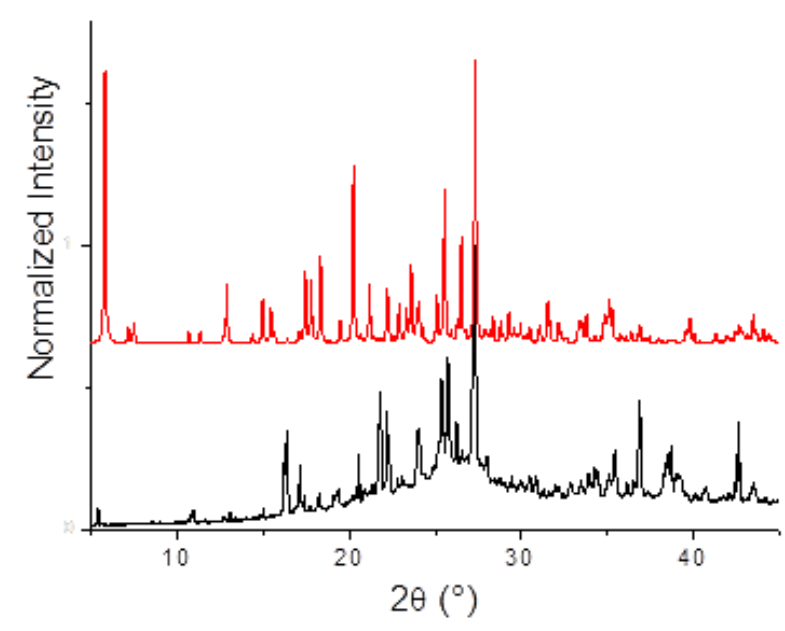

Figure 8. X-ray diffraction pattern of nanoparticles of $(\mathrm{BEDT}-\mathrm{TTF})_{2}\left[\mathrm{Cu}(\mathrm{SCN})_{2}\right]$ (black line) and pattern simulated from the single crystal structure of $\kappa$-(BEDT$\mathrm{TTF})_{2}\left[\mathrm{Cu}(\mathrm{SCN})_{2}\right]$ (red line).

$\underline{\text { Study of the solubility of NPs of organic }}$ conductors: one reason for growing materials as nanoparticles is to obtain them as solutions or stable suspensions. As single crystals, organic metals show, as metals, very low solubility in conventional solvents. Colloidal solutions of various metals are available and highly valuable in electronics or for catalysis applications [5]. The intrinsic chemical and physical properties of organic conductors make them of interest in the field of organic electronics. For instance, in OFET (Organic Field Effect Transistor) structures, electrical contacts are made between different organic materials and it has been observed that the structure similarity between the materials in contact is in favor of good electric transfer. For example, we have evaluated the use of stable suspensions of NPs of two organic conductors, TTF-TCNQ (TCNQ $=$ tetracyanoaquinodimethane) and TTF
$\left[\mathrm{Ni}(\mathrm{dmit})_{2}\right]_{2}(\mathrm{dmit}=$ dimercaptoisotrithione, or 1.3-dithio-2-thione-4,5-dithiolate), as contact electrodes in OFETs [19].

We investigated the solubility of the nanoparticle powders of $\mathrm{TTFCl}_{0.77}, \mathrm{TTFBr}_{0.59}$, (TMTSF) ${ }_{2} \mathrm{PF}_{6}$, BEDT-TTFCl ${ }_{0.66}$, and (BEDT$\mathrm{TTF})_{2}\left[\mathrm{Cu}(\mathrm{SCN})_{2}\right]$ in various solvents. Nanopowders of $\mathrm{TTFCl}_{0.77}, \mathrm{TTFBr}_{0.59}$, BEDT$\mathrm{TTFCl}_{0.66}$, and $(\mathrm{BEDT}-\mathrm{TTF})_{2}\left[\mathrm{Cu}(\mathrm{SCN})_{2}\right]$ are not soluble in acetone, acetonitrile or dichloromethane: the powders decant and the liquid remains uncolored. Addition of the nanopowder of (TMTSF) ${ }_{2} \mathrm{PF}_{6}$ to acetonitrile gives a yellow solution, best described as a stable suspension. Concentrations of up to 2.5 $\mathrm{mg} / \mathrm{mL}$ are obtained in acetonitrile. Bulk (TMTSF) $)_{2} \mathrm{PF}_{6}$ is known to be soluble in trichloroethane. However, the concentrations obtained from bulk dissolution [20] are 10 times lower than those obtained from nanoparticle powders: $0.2 \mathrm{mg} / \mathrm{mL}$ vs. $2.5 \mathrm{mg} / \mathrm{mL}$. It should be noted that the properties and shape of the nanoparticles are not modified after evaporation of the solvent [8].

\section{Conclusions}

We have explored the conditions of use of imidazolium or ammonium-based ionic liquids to favor the electrochemical growth of organic conductors as nanoparticles in the case of the five following phases: $\mathrm{TTFCl}_{0.77}$, $\mathrm{TTFBr}_{0.59}, \quad(\mathrm{TMTSF})_{2} \mathrm{PF}_{6}, \quad$ BEDT-TTFCl $_{0.66}$, (BEDT-TTF $)_{2} \mathrm{Br}$. The ionic liquid is used as the supporting electrolyte in the 
electrocrystallization process. The chemical the $\alpha$-phase. We have further studied the composition of the IL is adjusted as a function of the expected phase. The cation, a long chain ammonium or an imidazolium, is chosen to limit the growth of the crystallite and avoids the preparation of long needles or platelets. The anion is selected similar to the anion of the expected organic metal. The originality of these conditions is that only the chemicals necessary to reach the selected organic conductor are involved in the process. The IL cannot be used as the solvent because the electroactive organic molecule is not soluble enough. The same solvent as for electrocrystallization of single crystals is used. We have shown that the best conditions to readily obtain nanoparticles combine: (i) a $1 / 10$ ratio between the electroactive organic molecule and the IL, (ii) stirring of the anodic compartment to increase the contact of the electroactive species with the anode, and (iii) high current densities to maintain a constant growth rate during a minimum of time, the latter being important for producing large amounts of nanoparticle powders. The use of an IL as supporting electrolyte does not modify the composition and structure of the phases, but its presence is necessary for limiting the growth to nanoparticle size. We have then applied these conditions to prepare nanoparticles of the superconductor (BEDT-TTF $)_{2}\left[\mathrm{Cu}(\mathrm{SCN})_{2}\right]$. The isolated powder contains the $\kappa$-(BEDT$\mathrm{TTF})_{2}\left[\mathrm{Cu}(\mathrm{SCN})_{2}\right]$ phase in addition to possibly solubility of the isolated nanoparticle powders, an important characteristic for using them as conducting ink or paste in organic electronic devices. Among the nanopowders described here, only the (TMTSF) $)_{2} \mathrm{PF}_{6}$ ones exhibit a noticeable solubility and can be isolated as stable suspensions.

\section{Acknowledgements}

Benoit Cormary thanks the EURIPIDES project $\mathrm{N}^{\circ}$ EUR-11-125 for post-doctoral financial support.

\section{References}

[1] Valade L, Tanaka H. Molecular inorganic conductors and superconductors. In: Bruce DW, Walton R, editors. Molecular Materials. Contemporary Inorganic Materials book series. Chichester, UK: John Wiley \& Sons Ltd; 2010. p. 215-90.

[2] Cassoux P, Valade L, Fabre P-L. Electrochemical methods, electrocrystallisation. In: Lever ABP, editor. Comprehensive Coordination Chemistry II: From Biology to Nanotechnology, Fundamentals: ligands, Complexes, Synthesis, Purification and Structure. 1. Amsterdam: Elsevier; 2003. p. 761-73.

[3] Batail P, Boubekeur K, Fourmigué M, Gabriel J-CP. Electrocrystallization, an invaluable tool for the construction of ordered, electroactive molecular solids. Chem Mater. 1998;10(10):3005-15.

[4] Bönnemann H, Richards Ryan M. Nanoscopic metal particles - Synthetic methods and potential applications. Eur J Inorg Chem. 2001;2001(10):2455-80.

[5] Zheng W, Li D, Guo W. Applications of ionic liquids (ILs) in synthesis of inorganic nanomaterials. 2015. In: Ionic Liquids - Current State of the Art [Internet]. InTech; [93-117]. Available from: http://dx.doi.org/10.5772/59048. 
[6] de Caro D, Jacob K, Mazzi S, Carayon

M-T, Faulmann C, Valade L. First TTF-based conductor nanoparticles by electrocrystallization. Synth Met. 2012;162(9-10):805-7.

[7] de Caro D, Valade L, Faulmann C, Jacob K, Van Dorsselaer D, Chtioui I, Salmon L, Sabbar A, El Hajjaji S, Perez E, Franceschi S, Fraxedas J. Nanoparticles of molecule-based conductors. New J Chem. 2013;37(11):3331-6.

[8] de Caro D, Jacob K, Faulmann C, Valade L. First nanoparticles of Bechgaard salts. C R Chimie. 2013;16(7):629-33.

[9] Bozio R, Zanon I, Girlando A, Pecile C. Vibrational spectroscopy of molecular constituents of one-dimensional organic conductors. Tetrathiofulvalene (TTF), $\mathrm{TTF}^{+}$, and $\left(\mathrm{TTF}^{+}\right)_{2}$ dimer. J Chem Phys. 1979;71(5):2282-93.

[10] Meneghetti M, Bozio R, Zanon I, Pecile C, Ricotta C, Zanetti M. Vibrational behavior of molecular constituents of organic superconductors TMTSF, its radical cation and the sulfur analogs TMTTF and TMTTF+. J Chem Phys. 1984 1984;80(12):6210-24.

[11] Bozio R, Pecile C, Scott JC, Engler EM. Infrared and Raman-study of the anion-donor interactions in tetrahedral anion (TMTSF) $)_{2} \mathrm{X}$ and (TMTTF) ${ }_{2} \mathrm{X}$ salts. Mol Cryst Liq Cryst. 1985 1985;119(1-4):211-20.

[12] Wang HH, Ferraro JR, Williams JM, Geiser U, Schlueter JA. Rapid Raman spectroscopic determination of the stoichiometry of microscopic quantities of BEDT-TTF-based organic conductors and superconductors. J Chem Soc, Chem Commun. 1994;16:1893-4.

[13] Clavel G, Larionova J, Guari Y, Guerin C. Synthesis of cyano-bridged magnetic nanoparticles using room-temperature ionic liquids. Chem Eur J. 2006;12(14):3798-804.

[14] Becker JA, Schafer R, Festag R, Ruland W, Wendorff JH, Pebler J, Quaiser SA, Helbig W, Reetz MT. Electrochemical growth of superparamagnetic cobalt clusters. J Chem Phys. 1995;103(7):2520-7.

[15] de Caro D, Faulmann C, Valade L, Jacob K, Chtioui I, Foulal S, de Caro P, Bergez-Lacoste M, Fraxedas J, Ballesteros B, Brooks JS, Steven E, Winter LE. Four molecular superconductors isolated as nanoparticles. Eur J Inorg Chem. 2014;2014(24):4010-6.

[16] Urayama H, Yamochi H, Saito G, Nozawa K, Sugano T, Kinoshita M, Sato S, Oshima K, Kawamoto A, Tanaka J. A new ambient pressure organic superconductor based on BEDT-TTF with Tc higher than 10K (Tc=10.4K). Chem Lett. 1988;17(1):55-8.

[17] Gärtner S, Gogu E, Heinen I, Keller HJ, Klutz T, Schweitzer D. Superconductivity at $10 \mathrm{~K}$ and ambient pressure in the organic metal (BEDTTTF $)_{2} \mathrm{Cu}(\mathrm{NCS})_{2}$. Sol State Commun. 1988;65(12):15314.

[18] Kinoshita N, Takahashi K, Murata K, Tokumoto M, Anzai H. ESR and electrical properties of a new organic metal: $\alpha$-(BEDT-TTF $)_{2} \mathrm{Cu}(\mathrm{NCS})_{2}$. Solid State Commun. 1988;67(5):465-70.

[19] Kadoya T, de Caro D, Jacob K, Faulmann C, Valade L, Mori T. Charge injection from organic charge-transfer salts to organic semiconductors. J Mater Chem. 2011;21(45):18421-4.

[20] Angelova A, Moradpour A, AubanSenzier P. Solvent dependence of organic superconducting thin film formation. Synth Met. 2004;143(2):187-90. 